\title{
La estera andina como aislante para la rehabilitación energética de viviendas rurales en el páramo ecuatoriano.
}

The use of Andean mats as insulators in the energy rehabilitation of rural households in the Ecuadorian Paramo

Juan E. Ureña Moreno. ${ }^{1} \&$ Carlos Renato Chávez. ${ }^{2}$ \& Vicente Parra León ${ }^{3}$

\section{Abstract. \\ DOI: https://doi.org/10.33262/cienciadigital.v3i3.2.713}

The Andean mat, totora fiber made by artisans in the area, reveals its importance in the energy rehabilitation of the model of the Rural Housing Program where a series of houses have been built in the North Central Andean highlands by the Ministry of Urban Development and Housing (MIDUVI). These homes are built about 3000 meters above sea level in a particular ecosystem called "the Andean páramo", where indigenous native peoples of ancient traditions live. The Rural Housing Program presents a problem of habitability due to the low temperatures that occur in the Andean dawn, causing high percentages of abandonment of these homes by their beneficiaries.

The structure of the house was modeled in a specialized bioclimatic software called "ECOTECT ANALYSIS", using the previous knowledge of the materials and construction techniques that were used to build the house. This tool was linked to a climatic and sun orientation database "CLIMATE CONSULTANT 5.5" of free access through the University of California, Los Angeles (UCLA). The results of the analyzed

\footnotetext{
${ }^{1}$ Escuela Superior Politécnica de Chimborazo, Facultad de Recursos Naturales, Chimborazo, Ecuador, juan.urena@espoch.edu.ec

${ }^{2}$ Escuela Superior Politécnica de Chimborazo, Facultad de Recursos Naturales, Chimborazo, Ecuador, renato.chavez@espoch.edu.ec

${ }^{3}$ Escuela Superior Politécnica de Chimborazo, Facultad de Recursos Naturales, Chimborazo, Ecuador, vicente.parra@espoch.edu.ec
} 
area the" North Central Andean highlands ", reveal that the largest losses in the envelope are produced by the roof of fibercement in $24.6 \%$ and that these decrease to $5.3 \%$ if the mat is used as a suspended ceiling.

In order to determine the variations of the interior temperature, a meteorological station (WS1080) with a thermo-hydraulic transmitter was installed in a house of the Quinua Nam community tourism project built by MIDUVI, which is located at 3376above sea level in the Andean páramo of the Province of Chimborazo. More than 5000 readings were taken every half hour, as a result of this, it was determined that after the installation of the Andean mat, as a thermal insulator, the temperatures in the unfavorable early rises increased on average from 8 to 13 degrees Celsius. In conclusion, the use of the Andean mat is an economical solution of quick installation that turns the house into a more thermally comfortable place during the cold nights of the Paramo. This can be seen as an important detail that can make the different at the moment of thinking about abandoning these houses, as it is well known that there are more than 6,000 homes that are affected in the North Central Andean highlands of Ecuador.

Keywords: Envelop, Andean Mat, Paramo, Heat Gain, Rural Housing

\section{Resumen.}

La estera andina, fibra de totora confeccionada por los artesanos de la zona, devela su importancia en la rehabilitación energética para el modelo de vivienda en serie del programa de Vivienda Rural del Ministerio de Desarrollo Urbano y Vivienda (MIDUVI) enfocado en la Sierra Centro-Norte de los Andes Ecuatorianos. Dichas viviendas son construidas por encima de los 3000 metros sobre el nivel del mar en un ecosistema particular "el páramo andino" donde habitan indígenas originarios llenos de costumbres con una cosmovisión propia. El Programa de vivienda rural presenta un problema de habitabilidad debido a las bajas temperaturas que se presentan en la madrugada andina, provocando altos porcentajes de abandono de estas viviendas por parte de sus beneficiarios.

Se modeló la estructura de la vivienda en un software bioclimático especializado llamado "ECOTECT ANALYSIS", utilizando la información de los materiales y técnicas de construcción usadas. Esta herramienta fue enlazada a una base de datos climáticos y de orientación del sol "CLIMATE CONSULTANT 5.5" de acceso gratuito de la Universidad de California, Los Ángeles (UCLA). Para la zona en mención "la Sierra central ecuatoriana", los resultados del análisis revelan que las mayores pérdidas en la envolvente se producen por el techo de fibrocemento en un 24.6 $\%$ y que estas disminuyen a $5.3 \%$ si se usa la estera como techo falso.

Con el objetivo de determinar las variaciones de temperatura interior se instaló una estación meteorológica (WS1080) con transmisor termo hidráulico en una vivienda 
del proyecto de turismo comunitario Quinua Nam construida por el MIDUVI. Ubicada a 3376 msnm en el páramo andino de la Provincia de Chimborazo se tomaron más de 5000 lecturas en intervalos cada media hora, como resultado se determinó que después de la instalación de la estera en el techo como aislante térmico las temperaturas en las horas más desfavorables de la madrugada se incrementaron en promedio de 8 a 13 grados centígrados.

Se concluye que es una solución económica, de rápida instalación y que convierte a la vivienda en un lugar más confortable térmicamente en la fría noche de Paramo. Este hallazgo puede verse como un detalle importante que marca la diferencia entre el abandono de la estructura o su uso cotidiano lo cual es importante ya que se sabe que existen más de 6000 viviendas que están afectadas en la región central del Ecuador.

Palabras claves: Envolvente, Estera, Paramo, Ganancia Térmica, Vivienda Rural

\section{Introducción.}

Los Andes Ecuatorianos albergan una de las estructuras vernáculas de la herencia indígena "La Choza", semienterrada con una envolvente dotada de técnicas bioclimáticas intrínsecas, tiene un óptimo desempeño en el frío del páramo andino.

El déficit habitacional presentado por el MIDUVI, que para el año 2010 era de 692.216 unidades en todo el Ecuador, especifica que el 80 por ciento corresponde a familias de bajos ingresos. El gobierno del Ecuador con el objetivo de disminuir este déficit impulsó un programa de vivienda Rural en las comunidades de la Sierra Centro, muchos de estos cantones están catalogados con los más altos índices de pobreza de todo el país.

El Ministerio de Vivienda construyó un prototipo de vivienda que no toma en cuenta este ecosistema, donde existen saltos significativos de temperatura entre la noche y el día. El diseño propuesto descarta las técnicas constructivas tradicionales tales como la construcción en tierra o bahareque.

Más de 6000 viviendas de interés social fueron construidas y entregadas a beneficiarios en la Sierra Central del Ecuador. La población abandono su vivienda tradicional para habitar la "modernidad", sin embargo, a los pocos meses el porcentaje de abandono de las nuevas viviendas creció a tal punto que muchas de estas estructuras son usadas en la actualidad como bodegas de almacenamiento o protección de especies menores.

Apoyado en una experiencia previa de trabajo con estos pueblos y el conocimiento profundo de materiales, formas constructivas, clima y especificaciones; se plantea la hipótesis inicial; los materiales de la envolvente no están diseñados para este ecosistema. Convirtiéndose en objetivo primordial de este trabajo, someter la estructura del techo bajo un análisis bioclimático para demostrar el problema de habitabilidad por la falta de confort térmico que tienen estas unidades construidas en el páramo ecuatoriano. 
Las bajas temperaturas nocturnas son señaladas como la causa principal; en el Ecuador no existen investigaciones previas para el desempeño bioclimático en este tipo de estructuras para esta región; es por ello que esta investigación pretende brindar una solución rápida, de bajo costo y accesible a este segmento de la población. Las mejoras planteadas pretenden elevar la temperatura interior de las viviendas evitando así su abandono.

Las pruebas de campo ejecutadas en San Martin, Cantón Guamote, Provincia de Chimborazo, comunidad indígena que fue beneficiada con 14 viviendas del programa MIDUVI, se encuentra a más de 3600 metros sobre el nivel del mar en los Andes ecuatorianos

El análisis bioclimático próximo pretende reconocer toda la diversidad de factores a considerar y establece niveles de relación entre ellos suficientemente claros para proporcionar soluciones reales y adaptadas a la realidad social y económica de sus habitantes., para después realizar un aporte real con soluciones prácticas para el mejoramiento en su confort térmico con criterios pasivos, no descartando incluir algún sistema activo acorde a la realidad social y geográfica de la región o el uso de materiales específicos encontrados en el entorno próximo del páramo ecuatoriano.

\section{Metodologia.}

Este artículo de investigación detalla los resultados de una investigación aplicada que tiende a resolver un problema específico, la baja temperatura interior, con el desarrollo de una idea innovadora a corto y mediano plazo, el uso de la estera de totora.

El enfoque de la investigación aplicado a este objetivo fue cuantitativo, ya que se realizó una evaluación de comparación numérica de datos encontrados tanto en el software ECOTECT ANALYSIS como en la toma de lecturas con la estación meteorológica WS1080 instalada in situ, para los dos casos planteados, antes y después del uso de la estera como aislante.

Según Gómez, este enfoque usa la recopilación de información y su posterior análisis para responder interrogantes de investigación y probar hipótesis previamente establecidas (2006)

La metodología de este análisis pretende no perder de vista ningún aspecto parcial de la totalidad de lo analizado, poniendo en relación todos los factores a base de retroalimentación apoyado en un diagnostico ejecutado en un Software especializado que afirma la hipótesis de la falta de confort térmico en estas unidades habitacionales y muestran posibles caminos de intervención.

La modelación, bajo condiciones climáticas del páramo ecuatoriano y una orientación optima de la vivienda, oriento el mayor número de aberturas hacia el Este y Oeste; la Fig. 1.1 presenta las trayectorias solares sobre la estructura a lo largo del año. 
La trayectoria del sol en todo el año tiene una trayectoria de Oeste a este en la Sierra central del Ecuador, exponiendo al techo directa y verticalmente a los rayos solares la mayor parte del día, revelando así la importancia del techo como el elemento principal para la ganancia térmica en esta ubicación geográfica.

Fig. 1.1 Trayectoria Solar anual (Sierra Central Ecuador)

Fuente:

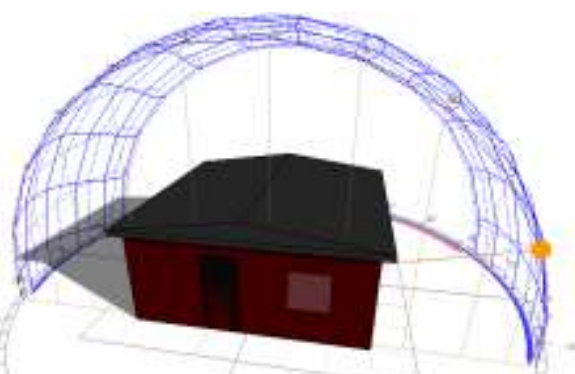

\section{Elaboración Propia}

ganancia térmica en horas distintas zonas de la La Figura 1.2 presenta la
de la tarde para las envolvente y sus valores totales recibidos en orientación óptima, la tabla adjunta demuestra
que la mayor ganancia en el día se produce en el techo, induciendo también la hipótesis que también tendrá la mayor perdida por la noche en esa zona de la envolvente.

Fig. 1.2 Valores totales de radiación envolvente en orientación óptima sierra central Ecuador
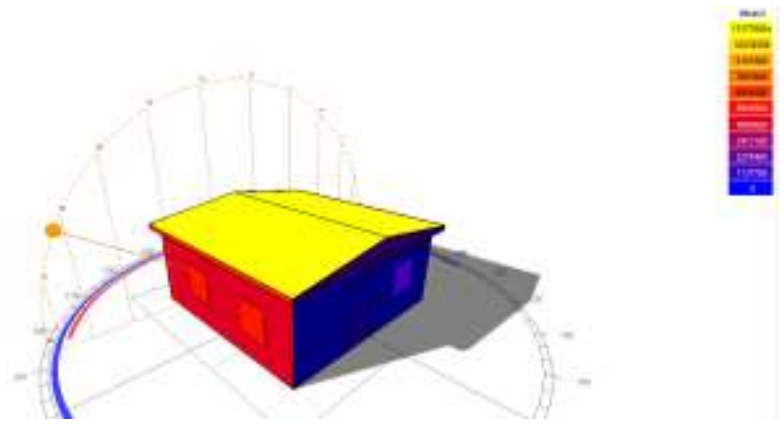

Fuente: elaboración propia

Develada la importancia del techo con respecto al sol para esta latitud, se hace un análisis de los materiales y su forma constructiva, especificaciones que se detallan a continuación:

Tiene una forma de techo a dos aguas y está fabricada en planchas de fibrocemento sujetas y asentadas sobre una estructura metálica soldada en el sitio (Fig. 1.3), no existen canales de recolección de aguas lluvias. 
MIDUVI detalla la cubierta con la siguiente normativa:

Cubierta

1 Correas metálicas De acuerdo con los planos y especificaciones, la estructura de la cubierta será con correas metálicas tipo G 80x40x15x1.5 ancladas en las cadenas superiores, debidamente recubiertas con pintura anticorrosiva.

2 Cubierta de De acuerdo con los planos y especificaciones, la cubierta será de fibrocemento. planchas de fibrocemento con una carga mínima en el ensayo de 1,83x1,10 m. flexión de $425 \mathrm{~kg}$ para $1 \mathrm{~m}$ de ancho por 6 pies, fijados con ganchos "J" galvanizados y capuchones. Para la instalación se tomará en cuenta los detalles señalados en los planos y las recomendaciones del fabricante. La unidad de medida será el $\mathrm{m} 2$ y el costo unitario el señalado en el contrato.

3 Caballetes de De acuerdo con los planos y especificaciones, los cumbreros de la fibrocemento cubierta será de fibrocemento, fijados con ganchos "J" galvanizados y capuchones. Para la instalación se tomará en cuenta los detalles señalados en los planos y las recomendaciones del fabricante. La unidad de medida será el ml y el costo unitario el señalado en el contrato.

Fuente: MIDUVI CHIMBORAZO

Fig. 1.3 Detalle constructivo Cubierta - Vivienda Rural MIDUVI CHIMBORAZO Comunidad Batzacon
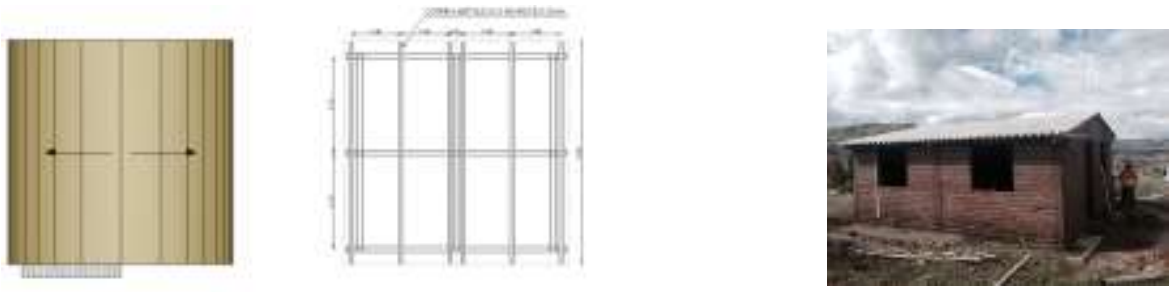

Fuente: Elaboración Propia

Conociendo los componentes del techo se determina el coeficiente de transmitancia térmica "K" $(\mathrm{K}=1 / \mathrm{R}) ; \mathrm{R}=(1 / \alpha 1+\mathrm{e} 1 / \lambda 1+1 / \alpha \mathrm{e})$ parámetro básico para la modelación de la envolvente. Dónde: e1/ $1=$ única cubierta (plancha asbesto cemento) $\alpha 1=$ Resistencia Superficial Interna $\alpha e=$ Capa de aire superficial externa 


\begin{tabular}{llll}
\hline $\begin{array}{l}\text { Capa de elemento } \\
\text { constructivo } \\
\text { (Techos) }\end{array}$ & $\begin{array}{l}\text { Espesor } \\
\text { e }(\mathrm{m})\end{array}$ & $\begin{array}{l}\text { Conductividad } \\
\text { térmica } \lambda(\mathrm{W} / \mathrm{mK})\end{array}$ & $\begin{array}{l}\text { Resistencia al paso } \\
\text { del calor } \\
\mathrm{R}\left(\mathrm{W} / \mathrm{m}^{2} \mathrm{~K}\right)\end{array}$ \\
\hline $\begin{array}{l}\text { Resistencia } \\
\text { superficial interna }\end{array}$ & & 0.17 \\
$\begin{array}{l}\text { Plancha asbesto } \\
\text { cemento Densidad }\end{array}$ & 0.010 & 0.87 & 0.012 \\
$\begin{array}{l}\text { aparente } 1300^{3} \\
\text { Capa de aire } \\
\text { superficial externa }\end{array}$ & 0.04 \\
Total & & 0.22 \\
\hline $\begin{array}{l}\text { El coeficiente de transmitancia térmica "K" para el techo es: } \mathrm{K}=1 / \mathrm{R} \mathrm{K}=1 / 0.22\left[\mathrm{~W} / \mathrm{m}^{2} \mathrm{~K}\right] \\
\mathrm{K}=4.54\left[\mathrm{~W} / \mathrm{m}^{2} \mathrm{~K}\right]\end{array}$
\end{tabular}

Fuente: Elaboración Propia

Con el uso del software bioclimático especializado "ECOTECT ANALYSIS", enlazado a la base de datos "CLIMATE CONSULTANT 5.5" para la zona sierra centro de los andes ecuatorianos. Se registran los valores obtenidos de transmitancia térmica para el techo, la fábrica de ladrillo y suelo; además de datos específicos de estimación de (Clo) para la vestimenta ancestral usada por los beneficiarios, horarios de habitabilidad y cocción de alimentos dentro de la estructura (Fig. 1.4). Obteniendo los siguientes resultados:

Fig. 1.4 (Izquierda) Valores para Condiciones Internas de Diseño, Ocupación y Operación), Derecha (Cronograma de ocupación de la estructura basado en experiencia en campo)
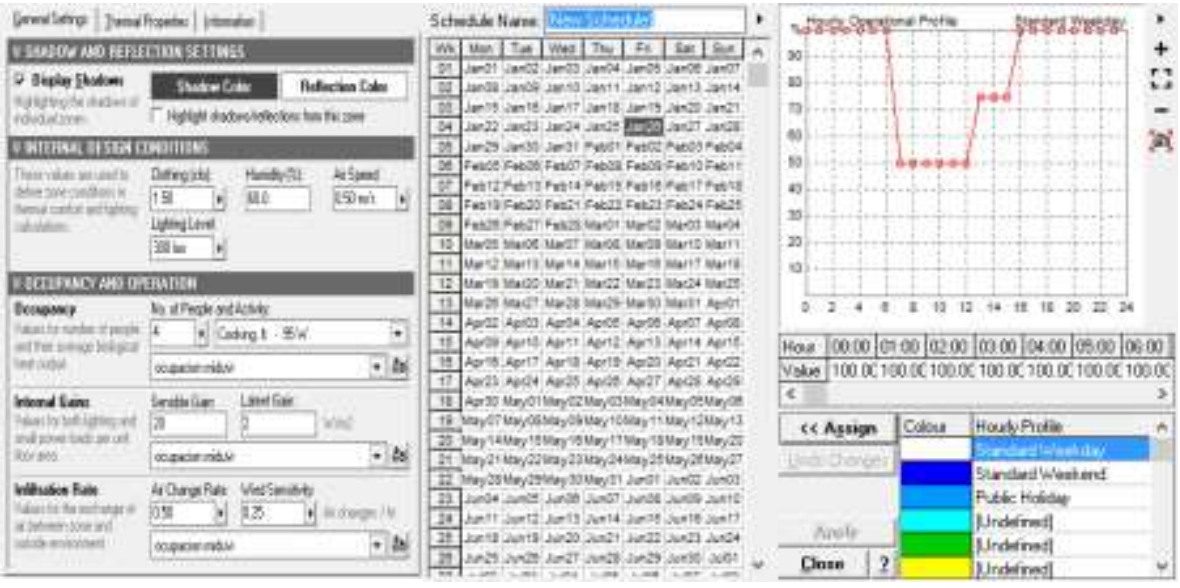

Fuente: Elaboración Propia

\section{Resultados.}


Al analizar el comportamiento de la cubierta, las perdidas representan el $24.6 \%$ del total por la falta de aislamiento.

Fig. 2.1 GANANCIAS Y PERDIDAS TECHO - Estructura del techo Vivienda MIDUVI

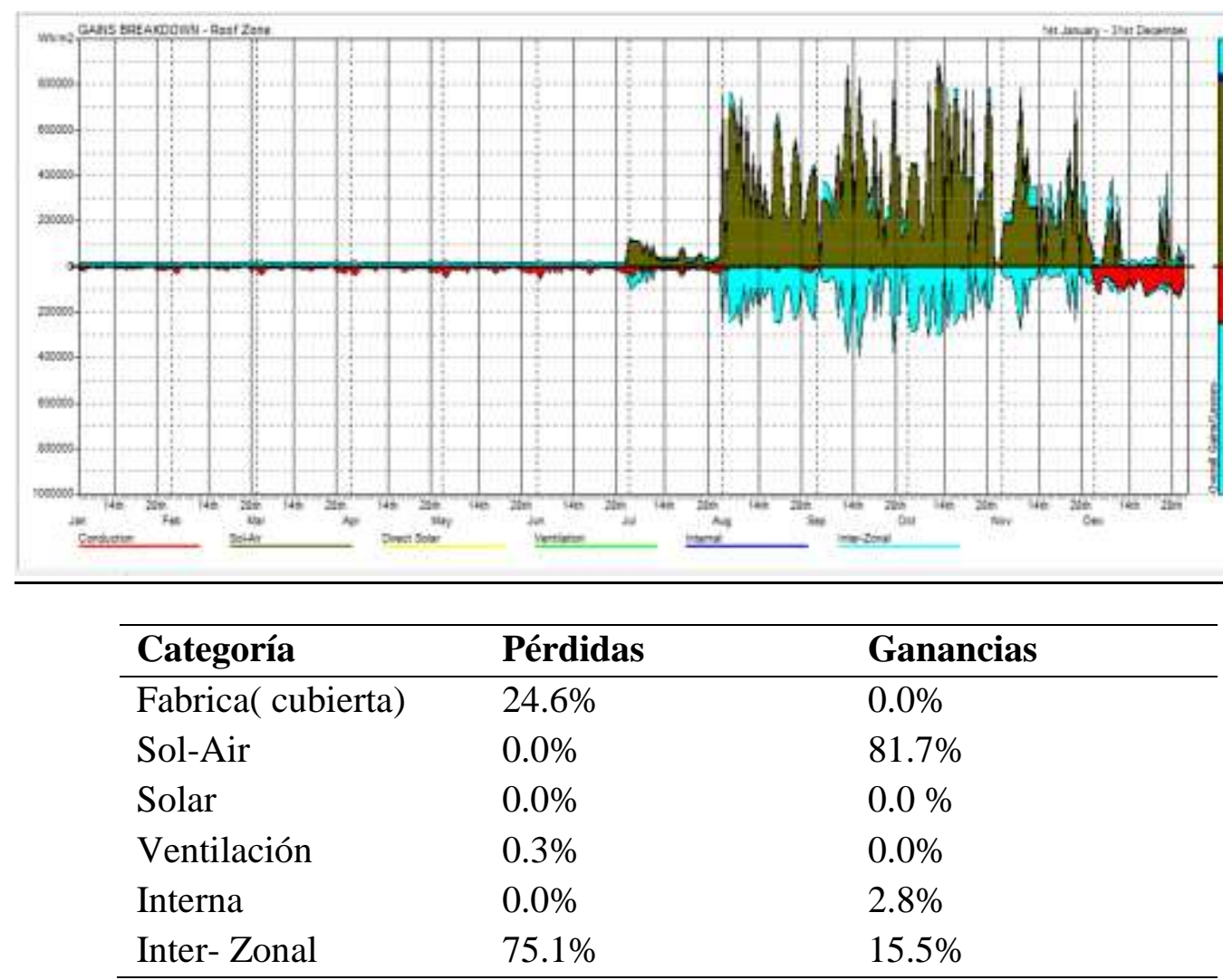

Fuente: ECOTECT ANALYSIS - Elaboración Propia

Demostrado que el techo de fibrocemento sin cámara de vacío tiene pérdidas del $24.6 \%$, se plantea el uso de un material como aislante, económico y de fácil acceso para el segmento de usuarios, la estera de totora artesanal tiene propiedades reconocidas y resistencia al paso del tiempo. Una estructura de madera de eucalipto apoyada sobre las paredes internas servirá de soporte para la fibra, sobre habitaciones y espacios comunes instalados a una altura de 2.20 metros desde el suelo. (Fig. 2.2)

Fig. 2.2 Detalle de instalación estera

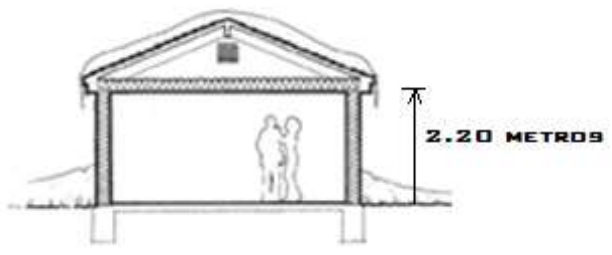

\section{Modelación con estera}


Simulando la cámara de aire y el tejido hecho de estera con valores de conductividad térmica de $0.041[\mathrm{~W} / \mathrm{m} \mathrm{K}]^{3}$ correspondientes a fibras de rápido crecimiento como el lino, la fibra de cáñamo o su equivalente "la totora", que producen fibras aptas para su uso como aislante, siendo ligera y con energía incorporada de entre 1228-1422 [W/Kg] se obtuvo que:

Las pérdidas por el techo se ubicaron en menos del 6\%. La Fig. 2.3 presenta en color rojo el valor referido.

Fig. 2.3 Ganancias y pérdidas techo después de intervención - Estructura de techo Vivienda MIDUVI

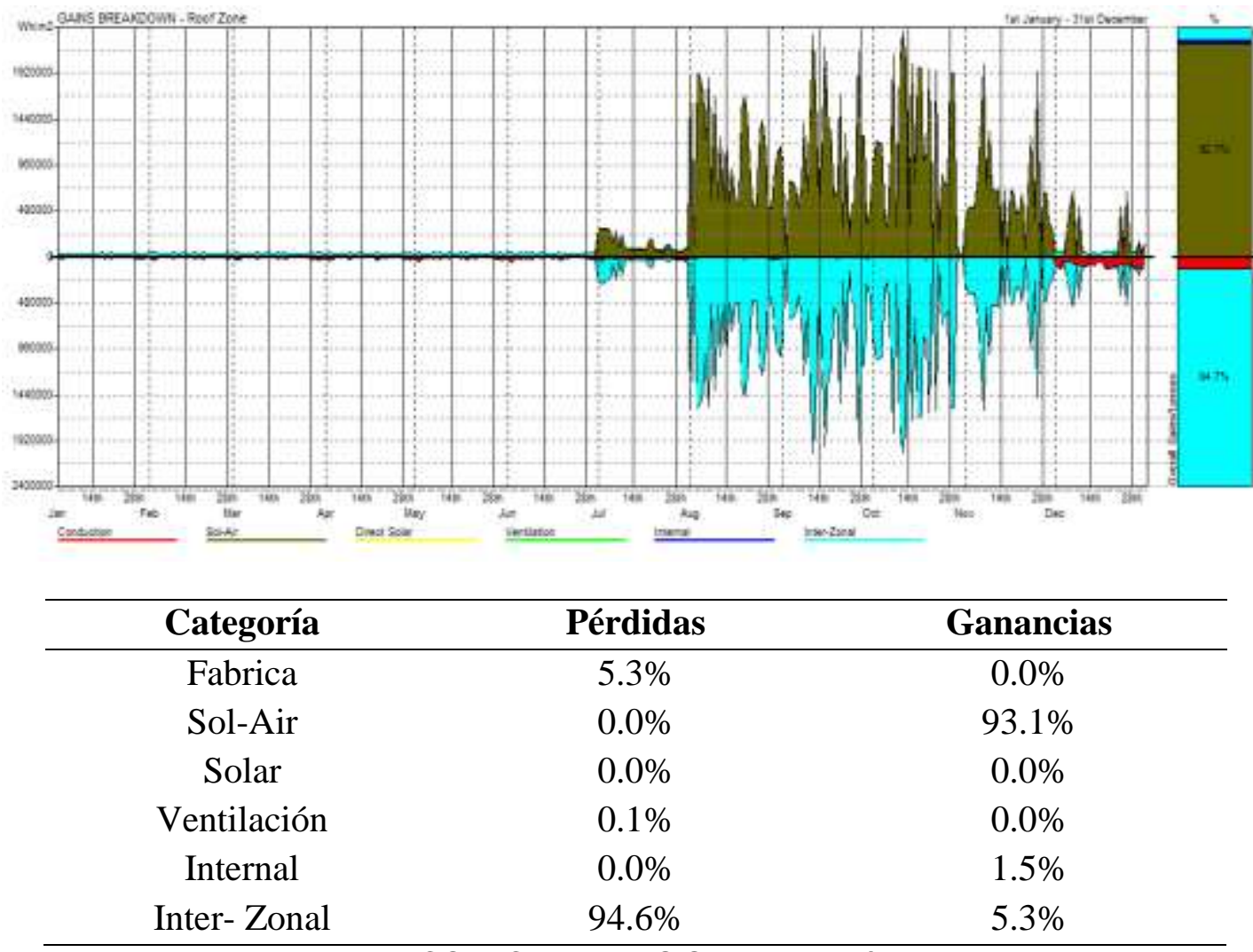

Fuente: ECOTECT ANALYSIS - Elaboración Propia

Las pérdidas por el techo disminuyeron desde $24.6 \%$ a menos de $6 \%$. La disminución más significativa en la envolvente después de la intervención.

Haciendo un análisis de la temperatura interior, el monitoreo con la estación meteorológica WS1080 se ejecutó antes de la intervención con estera por un periodo de 3 meses, para después continuar con el monitoreo durante 3 meses más con aislante de estera en el techo.

Las temperaturas menos favorables se dan en promedio entre las $4 \mathrm{~h} 00$ y $6 \mathrm{~h} 00$ horas en todos los periodos diarios con un promedio de 6 a 7 grados centígrados. (Fig. 2.4) y las máximas entre las $14 \mathrm{~h} 00$ y $15 \mathrm{~h} 00$ horas alcanzando 22 grados centígrados. 
Fig. 2.4 monitoreo temperatura interior vivienda sin intervención (Lecturas de la estación meteorológica)

\begin{tabular}{|c|c|c|}
\hline $\begin{array}{l}12.12 .2017 \\
23: 55: 34\end{array}$ & 30 & 9.7 \\
\hline $\begin{array}{l}13.12 .2017 \\
00: 25: 34\end{array}$ & 30 & 9.3 \\
\hline $\begin{array}{l}13.12 .2017 \\
00: 55: 34\end{array}$ & 30 & 9 \\
\hline $\begin{array}{l}13.12 .2017 \\
01: 25: 34\end{array}$ & 30 & 8.7 \\
\hline $\begin{array}{l}13.12 .2017 \\
01: 55: 34\end{array}$ & 30 & 8.4 \\
\hline $\begin{array}{l}13.12 .2017 \\
02: 25: 34\end{array}$ & 30 & 8 \\
\hline $\begin{array}{l}13.12 .2017 \\
02: 55: 34\end{array}$ & 30 & 7.5 \\
\hline $\begin{array}{l}13.12 .2017 \\
03: 25: 34\end{array}$ & 30 & 7.5 \\
\hline $\begin{array}{l}13.12 .2017 \\
03: 55: 34\end{array}$ & 30 & 7.1 \\
\hline $\begin{array}{l}13.12 .2017 \\
04: 25: 34\end{array}$ & 30 & 6.9 \\
\hline $\begin{array}{l}13.12 .2017 \\
04: 55: 34\end{array}$ & 30 & 6.6 \\
\hline $\begin{array}{l}13.12 .2017 \\
05: 25: 34\end{array}$ & 30 & 6.3 \\
\hline $\begin{array}{l}13.12 .2017 \\
05: 55: 34\end{array}$ & 30 & 6.1 \\
\hline $\begin{array}{l}13.12 .2017 \\
06: 25: 34\end{array}$ & 30 & 5.8 \\
\hline
\end{tabular}

Fuente: ESTACION METEREOLOGICA WS1080

Fig. 2.5 monitoreo temperatura interior vivienda sin intervención (Interpretación gráfica)

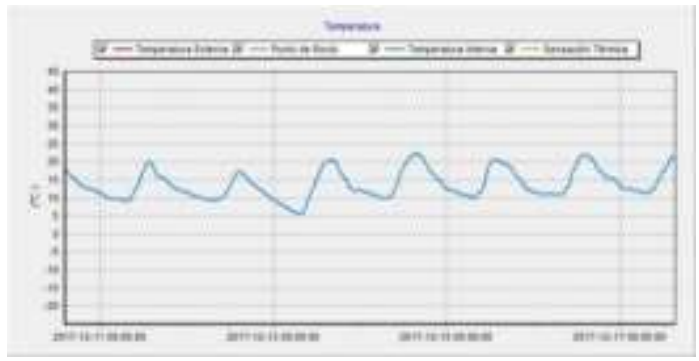

Fuente: ESTACION METEREOLOGICA WS1080 
Después de la intervención (Fig. 2.5) las temperaturas menos favorables continúan dándose entre las $4 \mathrm{~h} 00$ y $6 \mathrm{~h} 00$ horas en todos los periodos diarios pero con medias de 13 grados centígrados. (Fig. 2.6) y las máximas entre las $14 \mathrm{~h} 00$ y $15 \mathrm{~h} 00$ horas alcanzando 20 grados centígrados.

Fig. 2.6 ESTACION METEREOLOGICA WS1080 en vivienda intervenida con estera.
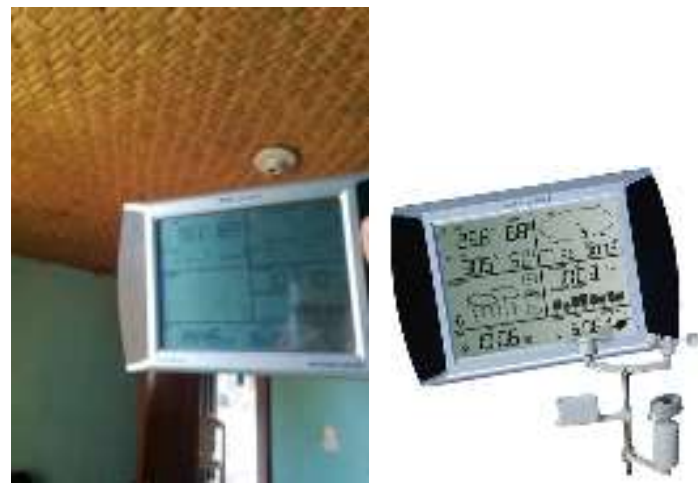

Fuente: Elaboración Propia

Fig. 2.7 monitoreo temperatura interior vivienda con intervención

\begin{tabular}{llc}
\hline $\begin{array}{l}21.05 .2018 \\
00: 05: 32\end{array}$ & 30 & 13.2 \\
\hline 21.05 .2018 & 30 & 13.1 \\
$00: 35: 32$ & & \\
21.05 .2018 & 30 & 13.1 \\
$01: 05: 32$ & & \\
21.05 .2018 & 30 & 13.1 \\
$01: 35: 32$ & & \\
21.05 .2018 & 30 & 13 \\
$02: 05: 32$ & & \\
21.05 .2018 & 30 & 13 \\
$02: 35: 32$ & & \\
21.05 .2018 & 30 & 13 \\
$03: 05: 32$ & & \\
21.05 .2018 & 30 & 13 \\
$03: 35: 32$ & & \\
21.05 .2018 & 30 & 12.9 \\
$04: 05: 32$ & & \\
21.05 .2018 & 30 & 12.8 \\
$04: 35: 32$ & & \\
21.05 .2018 & 30 & 12.7 \\
$05: 05: 32$ & & \\
\hline
\end{tabular}




\begin{tabular}{lll}
\hline 21.05 .2018 & 30 & 12.5 \\
$05: 35: 32$ & & \\
\hline
\end{tabular}

Fuente: ESTACION METEREOLOGICA WS1080 - Elaboración Propia

\section{Conclusiones.}

- Las mayores pérdidas en la envolvente se dan por el techo de fibrocemento con un $24.6 \%$ del total.

- El techo falso hecho de estera apoyado en una estructura de madera, disminuye las pérdidas por el techo del 24.6 al $5.3 \%$ de la ganancia total.

- Las temperaturas más bajas registradas se dan entre las 04h00 a 07h00 horas de la mañana con marcas de hasta 5 grados centígrados, sin embargo, las temperaturas máximas interiores bordean los 22 grados centígrados durante el día.

- El techo de fibrocemento con un coeficiente de transmitancia térmica de $\mathrm{K}=4.54$ $\left[\mathrm{W} / \mathrm{m}^{2} \mathrm{~K}\right]$, no protege a la vivienda de las bajas temperaturas de la madrugada andina, se propone vetar su uso para este piso climático, en el caso de que no use un techo falso como aislante.

- La estera como aislante en el techo aumenta la temperatura interior en un promedio de hasta 6 grados centígrados en las horas más frías de la madrugada.

- El bajo costo y fácil instalación, además de buenas características térmicas y fácil acceso al material por parte de los usuarios, hacen de la estera de totora la mejor opción como aislante térmico para la rehabilitación del techo.

- Se debe evitar pérdidas por espacios abiertos entre las ventanas de aluminio y la mampostería, las perdidas por espacios dejados en defectos constructivos hacen de las viviendas estancias frías.

- El suelo de la vivienda no debe usar materiales como piezas cerámicas o porcelanatos, ya que incrementan la sensación de frio en la estancia.

\section{Referencias bibliográficas.}

Aladren Acha, Neila Javier.(2014). Arquitectura Bioclimática y Construcción sostenible. DAPP Publicaciones Jurídicas SL.

Cena, Krzysztof, Clark, J. A., Politechnika Wroclawska. (1981). Bioengineering, thermal physiology, and comfort. Vol. 10. Amsterdam; New York: Elsevier Scientific Pub. Co.

Gonzalo, G \& Nota, V. (2003). Manual de arquitectura bioclimática. Buenos Aires, Argentina: Nobuko

González N. (2004). Arquitectura Bioclimática en un entorno Sostenible. Madrid: MunillaLería 
Neila González, Francisco J., and Bedoya Frutos, César. (1997). Técnicas arquitectónicas y constructivas de acondicionamiento ambiental. $2^{\mathrm{a}}$ ed. Vol. 1. Madrid: Munilla-Lería.

Olgyay, V. (2006). Arquitectura y Clima Manual de Diseño Bioclimático para Arquitectos y Urbanistas en un entorno Sostenible. Barcelona: Gustavo Gili.

Rozis J. \& Guinebault, A. (1997). Calefacción solar para regiones frías. Guía tecnológica para la aplicación de la vivienda y agricultura en países en desarrollo . Perú : Geres

Serra R. (2004). Arquitectura y Climas. Barcelona: Gustavo Gili. 


\section{PARA CITAR EL ARTÍCULO INDEXADO.}

Ureña Moreno, J. E., Renato Chávez, C., \& Parra León, V. (2019). La estera andina como aislante para la rehabilitación energética de viviendas rurales en el páramo ecuatoriano. Ciencia Digital, 3(3.2), 24-37. https://doi.org/10.33262/cienciadigital.v3i3.2.713

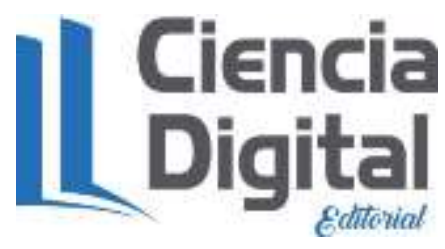

El artículo que se publica es de exclusiva responsabilidad de los autores y no necesariamente reflejan el pensamiento de la Revista Ciencia Digital.

El artículo queda en propiedad de la revista y, por tanto, su publicación parcial y/o total en otro medio tiene que ser autorizado por el director de la Revista Ciencia Digital.
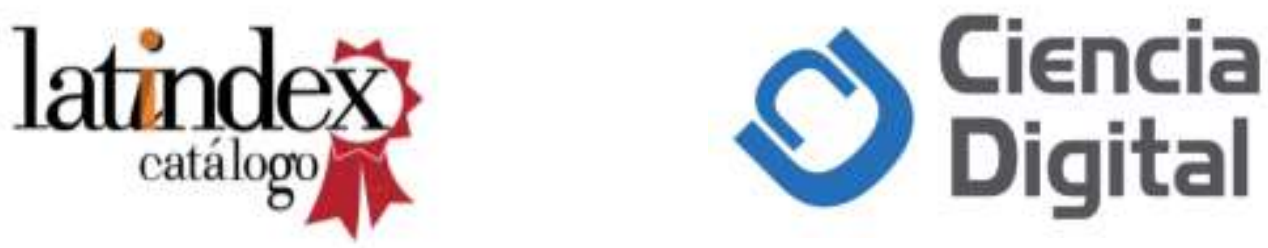